\title{
Tenuigenin promotes the osteogenic differentiation of bone mesenchymal stem cells in vitro and in vivo
}

\author{
Hua-ji Jiang ${ }^{1}$ - Xing-gui Tian ${ }^{1}$ - Shou-bin Huang ${ }^{2}$ - Guo-rong Chen ${ }^{1}$ • Min-jun Huang ${ }^{1}$ • \\ Yu-hui Chen ${ }^{1}$ - Bin Yan ${ }^{1}$. Sheng-fa $\mathrm{Li}^{2}$ • Jia-jun Tang ${ }^{1} \cdot$ Hui-yu Zhao ${ }^{1}$ Liang Wang ${ }^{1}$. \\ Zhong-min Zhang ${ }^{1}$
}

Received: 21 May 2016/Accepted: 7 October 2016/Published online: 14 November 2016

(C) The Author(s) 2016. This article is published with open access at Springerlink.com

\begin{abstract}
Osteoporosis, which is a systemic skeletal disease characterized by low bone mineral density and microarchitectural deterioration of bone quality, is a global and increasing public health problem. Recent studies have suggested that Tenuigenin (TEN), a class of native compounds with numerous biological activities such as anti-resorptive properties, exerts protective effects against postmenopausal bone loss. The present study aims to investigate the osteogenic effects of TEN on bone mesenchymal stem cells (BMSCs) in vitro and in vivo. Alkaline phosphatase (ALP) activity/staining, Alizarin red staining and the expression of osteogenic markers, including runt-related transcription factor 2 , osterix, osteocalcin, collagen $I \alpha 1, \beta$-catenin and glycogen synthase kinase- $3 \beta$ were investigated in primary femoral BMSCs from C57/BL6 mice cultured under osteogenic conditions for 2 weeks to examine the effects of TEN. An ovariectomized (OVX) mouse model was used to investigate the effect of TEN treatment for 3 months in vivo. We found that ALP activity, mineralized nodules and the expression of osteogenic markers were increased and $\mathrm{WNT} / \beta$-catenin signaling was enhanced in vitro and in vivo. Bone parameters, including trabecular thickness, trabecular number and bone mineral density were higher in
\end{abstract}

Hua-ji Jiang, Xing-gui Tian, Shou-bin Huang and Guo-rong Chen contributed equally to this work.

Liang Wang

sicot_china@163.com

Zhong-min Zhang

gukezhangzhongmin@163.com

1 Department of Orthopedics, The Third Affiliated Hospital, Southern Medical University, 183 West Zhongshan Avenue,

Guangzhou 510282, Guangdong, People's Republic of China

2 Department of Orthopedics, Huizhou First Hospital, Huizhou, Guangdong, People's Republic of China the OVX+TEN group than in control OVX mice. Our results suggest the therapeutic potential of TEN for the treatment of patients with postmenopausal osteoporosis.

Keywords Osteoporosis $\cdot$ Tenuigenin $\cdot$ BMSCs $\cdot$ Bone parameters $\cdot \mathrm{WNT} / \beta$-catenin signaling

\section{Introduction}

Osteoporosis (OP) is a common and systemic skeletal disease characterized by low bone mineral density (BMD) and microarchitectural deterioration of bone quality that reduces bone strength, with a consequent increased risk of fragile fractures (van den Bergh et al. 2012). More than 200 million people worldwide are affected by osteoporosis, especially postmenopausal women and older men (Liu et al. 2015; Tao et al. 2016). Osteoporosis increases the frequency of fractures of the hip, spine and wrist in association with substantial morbidity and mortality and the direct costs of osteoporotic treatments appear to be rising dramatically concomitant with the increase in life expectancy (Li et al. 2016). Although hormone replacement therapy is the most common therapeutic approach for the prevention and treatment of postmenopausal osteoporosis, the Women's Health Initiative reported that the health risks of hormone replacement therapy exceed its benefits (Yu et al. 2016). The use of bisphosphonates for the treatment of osteoporosis has also been reported (Bagan et al. 2016; Eriksen et al. 2014; Safer et al. 2016). However, the potential bone-forming agents in bisphosphonates are associated with serious side effects and may not yield the expected improvements in bone quality and bone union ratio (Luhe et al. 2008). Furthermore, the cost-effectiveness of their widespread or long-term use has been questioned. Despite the availability of an armamentarium of agents, finding the 
optimal agent remains a challenge (Demontiero et al. 2012). Therefore, it is desirable to identify better and safe anabolic agents for the treatment of osteoporosis.

Bone mass is controlled by continuous bone remodeling through osteoblastic bone formation and osteoclastic bone resorption. Abnormalities in bone remodeling can produce a variety of bone-decreasing disorders such as osteoporosis (Rodan and Martin 2000). During bone remodeling, the removal of old bone from the skeleton is performed by osteoclasts, which are derived from hematopoietic stem cells and the addition of new bone occurs through differentiation/ mineralization by osteoblasts, which are derived from mesenchymal stem cells (Boyle et al. 2003; Harada and Rodan 2003). If the balance between bone formation and bone resorption is disturbed and the rate of bone formation is lower than that of bone resorption, adult skeletal diseases can result in osteoporosis.

Bone mesenchymal stem cells (BMSCs) are composed of progenitor and multipotent skeletal stem cells and can differentiate into osteoblasts, osteocytes, adipocytes and chondro cytes in vitro (Ma et al. 2015). At present, it is understood that all osteoblasts are derived from BMSCs (Zhang et al. 2016). As BMSCs can differentiate into skeletal cell phenotypes (Bianco et al. 2006), they are the most promising and thoroughly investigated population of stem cells in osteoporosis research.

Tenuigenin (TEN), the chemical structure of which has been elucidated, is the active component of the root of the Chinese herb Polygala tenuifolia. It has a variety of biological activities, including anti-apoptotic, anti-oxidative and antiinflammatory effects (Liang et al. 2011; Sun et al. 2007). A recent report suggested that TEN is a potential drug for the treatment of osteoporosis by suppressing osteoclastogenesis (Yang et al. 2015). Osteoblast differentiation and osteoclastogenesis are two important phases of bone remodeling; however, whether TEN affects the osteogenic differentiation of BMSCs in vitro and bone formation in vivo remains unknown.

In the present study, we investigate the osteogenic activity of TEN and its underlying mechanism in vitro and in vivo. Our results indicate that TEN induces the differentiation of BMSCs into osteoblasts by activating $\mathrm{WNT} / \beta$-catenin signaling.

\section{Materials and methods}

\section{TEN preparation}

TEN (Fig. 1a) was obtained from Sigma-Aldrich (St Louis, MO, USA). A stock solution of TEN $(10 \mathrm{mg} / \mathrm{ml})$ was prepared in dimethyl sulfoxide (DMSO; Sigma-Aldrich) and stored at $2-8{ }^{\circ} \mathrm{C}$. TEN stock was diluted in sterile phosphate-buffered saline (PBS) and PBS-DMSO (0.16 \%) served as the control in cell culture experiments.

\section{Animals and drug treatment}

A total of 30 female C57BL/6 mice aged 8 weeks and weighing $22 \pm 3 \mathrm{~g}$ were provided by the Southern Medical University (Guangzhou, Guangdong, People's Republic of China). The mice were assigned equally to each of three groups: sham, ovariectomized (OVX) and OVX+TEN. OVX+TEN mice received TEN $(6 \mathrm{mg} / \mathrm{kg} /$ day $)$ intragastrically every day for 3 months after ovariectomy. The sham and OVX groups received the same amount of normal saline. Ethical approval for this study was obtained from the Medical Ethics Committee of the Southern Medical University (2015-095). The mice were kept following the animal care guidelines of the Southern Medical University laboratory animal welfare and ethics committee charter.

\section{Cell culture and osteogenic differentiation}

BMSCs were isolated from C57BL/6 mice (aged 4 weeks). Briefly, mice femurs were dissected free of surrounding soft tissue. The bone marrow was flushed with $\alpha$-MEM (Gibco, Grand Island, NY, USA). The marrow content from four to six bones was plated in culture flasks containing BMSC growth media ( $\alpha$-MEM containing $10 \%$ fetal bovine serum, $100 \mathrm{U} / \mathrm{ml}$ penicillin, $100 \mathrm{mg} / \mathrm{ml}$ streptomycin sulfate; Sigma-Aldrich). Non-adherent cells were removed and adherent BMSCs were cultured and expanded for further experiments. Primary cells were used in the experiments prior to the third passage. Cell culture media were replaced every 3 days.

\section{Cell proliferation assay}

Primary BMSCs were seeded in 96-well plates at a density of $3.13 \times 10^{4}$ cells $/ \mathrm{cm}^{2}$. After 2 days in culture, cells were treated with TEN at concentrations of $4,8,16$ and $32 \mu \mathrm{g} / \mathrm{ml}$ for 14 days. Cell proliferation was assessed using the Cell Counting Kit-8 (CCK-8; Keygen Biotech, Nanjing, People's Republic of China) colorimetric assay according to the manufacturer's instructions. Absorbance was measured at $450 \mathrm{~nm}$.

\section{ALP activity/staining assay}

Cells were plated into six-well plates at a density of $2.08 \times 10^{4}$ cells $/ \mathrm{cm}^{2}$. Following treatment with TEN $(4-16 \mu \mathrm{g} / \mathrm{ml})$ and osteoblast differentiation for 14 days, the cells were washed twice with PBS, scraped into $500 \mu \mathrm{L}$ of $10 \mathrm{mM}$ Tris-HCl buffer (pH 7.6) containing $0.1 \%$ Triton $\mathrm{X}-100$, placed on ice and sonicated to lyse the cells. Protein concentrations in the lysates were 
a
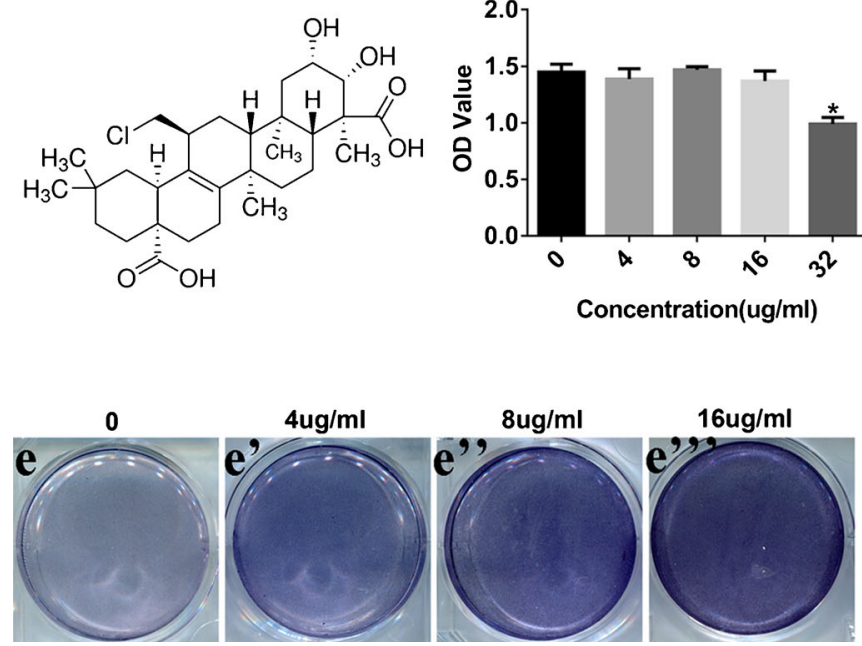

Fig. 1 TEN increased osteogenesis in BMSCs. a Structure of TEN. b BMSCs was treated with various concentrations of TEN $(4,8,16 \mu \mathrm{g} / \mathrm{ml})$ for 14 days and cell viability was measured by using the CCK-8 assay. BMSCs were incubated with TEN $(4,8,16 \mu \mathrm{g} / \mathrm{ml})$ in osteogenic differentiation medium for 14 days, then ALP activity (c), mineralized

determined using the Bradford protein assay. Alkaline phosphatase (ALP) activity in the cellular fraction was measured using a fluorometric detection kit (Nanjing Jiancheng Biotechnology, Nanjing, People's Republic of China). A standard curve was created using $p$-nitrophenol as the standard and the ALP activity of each sample was calculated from optical density $(450 \mathrm{~nm})$ values. Parallel wells were stained for ALP. Cells were fixed in $4 \%$ paraformaldehyde (Sigma-Aldrich) for $20 \mathrm{~min}$ at room temperature, washed, incubated with ALP staining buffer (NBT-BCIP; Sigma-Aldrich) at $37{ }^{\circ} \mathrm{C}$ for $30 \mathrm{~min}$ and washed with $\mathrm{PBS}$ to remove excess dye.

\section{Alizarin red assay}

BMSCs $\left(2.08 \times 10^{4}\right.$ cells $\left./ \mathrm{cm}^{2}\right)$ were plated and cultured in six-well plates with various concentrations of TEN $(4-16 \mu \mathrm{g} / \mathrm{ml})$ in osteogenic medium for 14 days. On day 14, the Alizarin red assay (Sigma-Aldrich) was performed to determine mineralization. Briefly, cells were washed with PBS and fixed with $4 \%$ paraformaldehyde for $30 \mathrm{~min}$ at room temperature. Fixed cultures were incubated with $1 \%$ Alizarin red for $30 \mathrm{~min}$ and washed with PBS to remove excess dye. Extracellular matrix mineral-bound stain was photographed under a microscope. For quantification, the bound staining was eluted with $10 \%$ (wt/vol) cetylpyridinium chloride (Sigma-Aldrich) and the absorbance of the supernatants was measured using an automated microplate reader (Bio-Rad, Hercules, CA, USA) at $570 \mathrm{~nm}$.
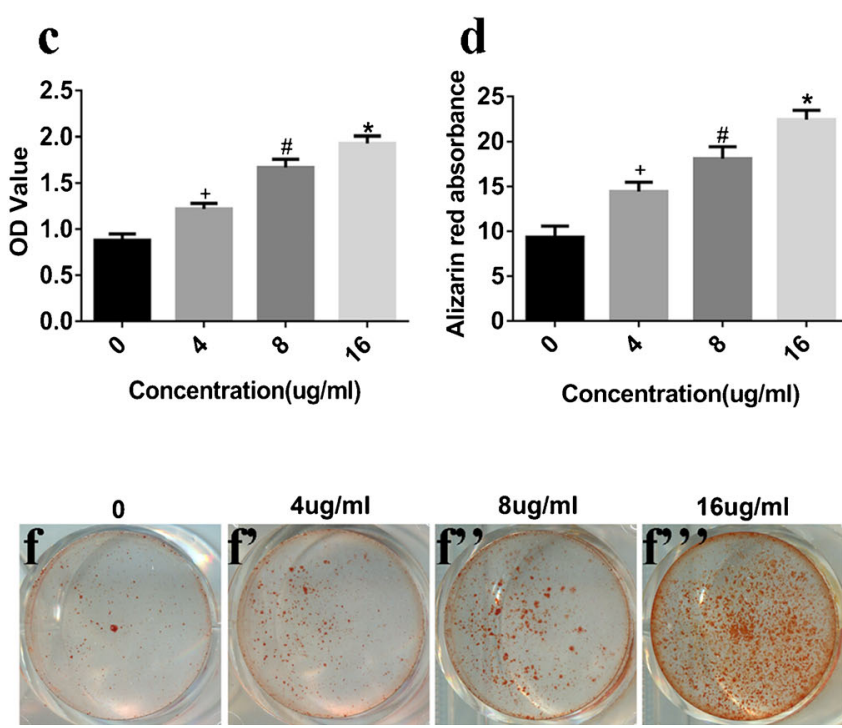

nodules (d), ALP staining (e-e'"), and Alizarin red staining (f- $-\mathbf{f}$ "') were tested. ${ }^{+} P<0.05$ versus group without TEN, ${ }^{\#} P<0.05$ compared with $4 \mu \mathrm{g} / \mathrm{ml} \mathrm{TEN}, * P<0.05$ compared with $8 \mu \mathrm{g} / \mathrm{ml} \mathrm{TEN}$. Columns represent means \pm SD from 3 independent experiments, each performed in triplicate

\section{Quantitative reverse transcription PCR (qRT-PCR) assay}

Total RNA was isolated from BMSCs with the TRIzol reagent (Life Technologies, Grand Island, NY, USA) according to the manufacturer's instructions after 2 weeks of osteogenic induction. Total RNA products were immediately converted to cDNA by reverse transcription (RT) using a PrimeScript RT reagent Kit with gDNA Eraser (TaKaRa, Dalian, China). Polymerase chain reaction (PCR) amplification was performed in a Chromo4 Four-Color Real-Time PCR Detection System (Bio-Rad) using a SYBRR Premix Ex Taq II (Tli RNaseH Plus) kit (TaKaRa). Primer sequences (Life Technologies) for each gene used in this study are shown in Table 1.

\section{Western blot (WB) assay}

Proteins isolated from six-well plates were subjected to SDS-polyacrylamide gel electrophoresis and transferred to polyvinylidene (PVDF) membranes (Sigma-Aldrich). Membranes were probed with rabbit polyclonal antibodies to runt-related transcription factor 2 (Runx2) (Cell Signaling Technology, Danvers, MA, USA), osterix (OSX) (Cell Signaling Technology), osteocalcin (OCN) (Santa Cruz Biotechnology, Santa Cruz, CA, USA), $\beta$ actin (Santa Cruz Biotechnology), $\beta$-catenin (Santa Cruz Biotechnology), glycogen synthase kinase-3 $\beta$ (GSK-3 $\beta$ ) (Santa Cruz Biotechnology) and goat anti-rabbit secondary antibody (Santa Cruz Biotechnology). PVDF membranes were incubated with primary antibodies for $8 \mathrm{~h}$ at $4{ }^{\circ} \mathrm{C}$ and washed three times with TRIS-buffered 
Table 1 Primer sequences used in RT-PCR and qPCR

\begin{tabular}{|c|c|c|}
\hline Target gene & GenBank accession no. & Sequences $\left(5^{\prime}-3^{\prime}\right)$ \\
\hline \multirow[t]{2}{*}{ GAPDH } & \multirow[t]{2}{*}{ NM_017008.4 } & Forward, CAGGGCTGCCTTCTCTTGTG \\
\hline & & Reverse, GATGGTGATGGGTTTCCCGT \\
\hline \multirow[t]{2}{*}{ RUNX2 } & \multirow[t]{2}{*}{ NM_001145920.2 } & Forward, AATTAACGCCAGTCGGAGCA \\
\hline & & Reverse, CACTTCTCGGTCTGACGACG \\
\hline \multirow[t]{2}{*}{ OSX } & \multirow[t]{2}{*}{ NM_001037632.1 } & Forward, GCCTACTTACCCGTCTGACTTT \\
\hline & & Reverse, GCCCACTATTGCCAACTGC \\
\hline \multirow[t]{2}{*}{$\mathrm{OCN}$} & \multirow[t]{2}{*}{ NM_001037939.2 } & Forward, TCTATGACCTGCAGAGGGCT \\
\hline & & reverse,ATAGCTCGTCACAAGCAGGG; \\
\hline \multirow[t]{2}{*}{ Coll $\alpha 1$} & \multirow[t]{2}{*}{ NM_000088.3 } & Forward, AGTGGTTTGGATGGTGCCAA \\
\hline & & Reverse, GCACCATCATTTCCACGAGC \\
\hline \multirow[t]{2}{*}{$\beta$-catenin } & \multirow[t]{2}{*}{ XM_006511927.1 } & Forward, CTGCAACGACCTGACTGGTA \\
\hline & & Reverse, GGCCATGTCCAACTCCATCA \\
\hline \multirow[t]{2}{*}{ GSK-3 $\beta$} & \multirow[t]{2}{*}{ XM_006522425.1 } & Forward, AGAAGAGCCATCATGTCGGG \\
\hline & & Reverse, CCAAAAGCTGAAGGCTGCTG \\
\hline
\end{tabular}

saline with Triton X-100 (TBST; 5 min per wash). The secondary antibody was incubated with the membranes for $1 \mathrm{~h}$ at room temperature, followed by three washes with TBST (5 min per wash). Signals were revealed using an enhanced chemiluminescence kit (Cell Signaling Technology).

\section{Immunofluorescence staining}

Cells were seeded into six-well plates containing glass cover slides at a density of $2.08 \times 10^{4}$ cells $/ \mathrm{cm}^{2}$ and grown to $95 \%$ confluence. The cells received osteoblast differentiation medium containing TEN for 2 weeks. Following fixation in $4 \%$ paraformaldehyde at room temperature for $30 \mathrm{~min}$, the cells were washed three times with PBST (0.1 \% Triton X-100 in PBS) and blocked in PBS-bovine serum albumin (1\% bovine serum albumin in PBS) for $1 \mathrm{~h}$ at room temperature. Cells were incubated with antibodies against collagen $\mathrm{I} \alpha 1$ (Col I $\alpha 1$ ) (Abcam, Cambridge, MA, USA) for $1 \mathrm{~h}$ at room temperature. The samples were then washed three times in PBS for $10 \mathrm{~min}$ each and incubated with antirabbit immunoglobulin $\mathrm{G}(\mathrm{H}+\mathrm{L}), \mathrm{F}(\mathrm{ab}$ ') 2 Fragment Alexa Fluor 594 conjugated secondary antibodies (Cell Signaling Technology) for $1 \mathrm{~h}$ at room temperature. For nuclear staining, cells were counterstained with 4',6diamidino-2 phenylindole (DAPI, Cell Signaling Technology) for $5 \mathrm{~min}$. Cells were examined using a laser confocal microscope (FV1000; Olympus Optical, Tokyo, Japan). Positive cells were evaluated using Image-Pro Plus software (Media Cybernetics, Rockville, MD, USA) to measure cellular fluorescence intensity. Cells with a fluorescence intensity $\geq 150 \%$ of background were considered positive.

\section{Histological and immunohistochemical assay}

Femur tissues dissected from the mice were fixed using $4 \%$ paraformaldehyde in PBS at $4{ }^{\circ} \mathrm{C}$ for $24 \mathrm{~h}$ and then decalcified in $15 \%$ ethylenediaminetetra-acetic acid $\left(\mathrm{pH} \mathrm{7.2)}\right.$ at $4{ }^{\circ} \mathrm{C}$ for 14 days. The tissues were embedded in paraffin or optimal cutting temperature compound (Sakura Finetek) and sectioned at 2-5 $\mu \mathrm{m}$. For histological analysis, the samples were stained with hematoxylin and eosin (HE) (Sigma-Aldrich). For immunohistochemistry, the sections were deparaffinized and briefly washed with PBS. This step was followed by incubation for $30 \mathrm{~min}$ in $3 \% \mathrm{H}_{2} \mathrm{O}_{2}$ to quench endogenous peroxidase activity. Appropriate primary antibodies anti-Runx2 (Cell Signaling Technology), anti-OSX (Cell Signaling Technology) and anti-OCN (Santa Cruz Biotechnology) were then applied overnight at $4{ }^{\circ} \mathrm{C}$. After incubation with the primary antibody, sections were washed three times with PBS and incubated with goat-anti-mouse horseradish peroxidaseconjugated secondary antibodies (Cell Signaling Technology) for $1 \mathrm{~h}$ at room temperature. Immunostained sections were then incubated with DAB. Finally, the sections were dehydrated, mounted with coverslips and examined using an Olympus light microscope.

\section{X-ray micro CT assay}

Long bones were collected from the mice, dissected free of soft tissue, fixed overnight in $4 \%$ paraformaldehyde and analyzed by high-resolution micro-computed tomography ( $\mu$ CT 80; Scanco Medical, Bruttisellen, Zurich, Switzerland). We set the scanner at a voltage of $89 \mathrm{kV}$, a current of $112 \mu \mathrm{A}$ and a scan thickness of $20 \mu \mathrm{m}$. Crosssectional images of the proximal tibiae and femora were acquired to perform three-dimensional histomorphometric 
analyses of the trabecular bone. The analyses included various bone parameters, including trabecular thickness, trabecular number and trabecular BMD.

\section{Statistical analysis}

Data are representative of at least three independent experiments. Each experiment was performed in triplicate. Data were graphed using GraphPad Prism software v.3.0. Oneway analysis of variance followed by the Student's $t$ test was used to determine statistical differences. Error bars represent the standard error of the mean in the cell experiments and the standard deviation in the animal experiments.

\section{Results}

\section{TEN has no toxic effects on the proliferation of BMSCs}

Firstly, the toxicity of TEN was measured by the CCK-8 assay. BMSCs were treated with different concentrations of TEN (4-, 8-, 16- and $32 \mu \mathrm{g} / \mathrm{ml}$ ) for 14 days. No alteration of cell viability was observed in BMSCs cultured in the presence of TEN at concentrations of 4,8 and $16 \mu \mathrm{g} / \mathrm{ml}$ (Fig. 1b). The test suggested that TEN is not harmful to the proliferation of BMSCs at concentrations of 4,8 and $16 \mu \mathrm{g} / \mathrm{ml}$.

\section{TEN stimulates osteogenic differentiation of BMSCs in vitro}

To examine the effects of TEN on osteogenic differentiation, BMSCs were treated with TEN $(4,8$ and $16 \mu \mathrm{g} / \mathrm{ml})$ in osteogenic medium for 14 days. Then, ALP activity/staining and Alizarin red staining were measured and the expression of the osteogenic markers Runx2, OSX, OCN and Col $\mathrm{I} \alpha 1$ was determined by qRT-PCR, WB, or immunostaining. TEN increased the expression of ALP (Fig. 1c, e-e"'), while mineralized nodules (Fig. 1d) and Alizarin red staining (Fig. 1f-f"') were also improved in a dose-dependent manner. The mRNA expression of Runx2 (Fig. 2a), OSX (Fig. 2b), OCN (Fig. 2c) and $\mathrm{Col} \mathrm{I} \alpha 1$ (Fig. 2d) was upregulated in a dose-dependent manner, as shown by qRT-PCR. In addition, the protein expression of Runx2 (Fig. 2e, f), OSX (Fig. 2e, g) and OCN (Fig. 2e, h) was also increased in a dose-dependent manner. Immunostaining for Col $\mathrm{I} \alpha 1$ (Fig. 2i-i"', j) showed a dosedependent increase that peaked at $16 \mu \mathrm{g} / \mathrm{ml}$. These results suggest that TEN promotes in vitro osteogenesis.

\section{Effect of TEN on femur bone microstructure morphometrics and histologic appearance}

To confirm the effect of ovariectomy, body weight and the mass of the uterus were recorded. Body weight increased in the OVX and OVX+TEN groups (Fig. 3d). The mass of the uterus in the sham group was significantly greater than that in the OVX group or OVX+TEN group (Fig. 3e). These data confirm that the excision of the ovaries was successful. To evaluate the effect of TEN on bone formation in mice, we performed X-ray $\mu \mathrm{CT}$ analyses of sham, OVX and OVX+TEN mice. X-ray $\mu \mathrm{CT}$ results for the long bones of the OVX+TEN mice demonstrated a clear trend in which trabecular thickness was increased relative to that in the OVX group (Fig. 3b-b", c-c", g). Trabecular number (Fig. 3b-b", c-c", f) and trabecular BMD (Fig. 3b-b", c-c", h) in bones from the OVX+TEN group were significantly higher than those in bones from the OVX group. However, these parameters were not significantly different between the OVX and sham groups. HE staining of femoral bone sections indicated that the number of trabeculae was significantly lower in OVX mice than in the OVX+TEN group (Fig. 3a-a").

\section{TEN promotes the expression of Runx2, OSX and OCN} in vivo

To examine the molecular and cellular changes associated with TEN-mediated protection against bone loss in vivo, we examined the epiphyseal growth plate, where bone formation occurs on a cartilaginous template (Karsenty 2003; Kronenberg 2003). Expression of Runx2 (Fig. 4a-a", d), OSX (Fig. 4b-b", e) and OCN (Fig. 4c-c", f) was markedly lower in OVX mice than in sham and OVX+TEN mice. However, there were no statistically significant differences between the sham group and the OVX+TEN group. Our in vitro and in vivo data indicate that TEN-induced osteogenesis positively regulates bone formation.

\section{Effect of TEN on WNT/ $\beta$-catenin signaling}

To gain further insight into the function of TEN in the osteogenic differentiation of cultured BMSCs, we examined the mRNA expression of $\beta$-catenin and GSK- $3 \beta$ in vitro. Our qRT-PCR results showed that $\beta$-catenin (Fig. 5a) and GSK$3 \beta$ (Fig. 5b) expression was enhanced. In addition, we investigated the $\beta$-catenin (Fig. 5 c, d) and GSK-3 $\beta$ (Fig. 5c, e) protein levels, which were both increased in BMSCs after TEN treatment. Collectively, these data indicate that WNT/ $\beta$-catenin signaling is increased in BMSCs in the presence of TEN and suggest that TEN cooperates with $\mathrm{WNT} / \beta$ catenin signaling to regulate bone formation.

\section{Discussion}

TEN is the active component of the Chinese herb Polygala tenuifolia root that has no genotoxic effects and is safe at the proper dose (Shin et al. 2015). It has been suggested as a 
potential drug for the treatment of osteoporosis (Yang et al. 2015). TEN also belongs to the family of saponin compounds, including ginsenoside, asperosaponin VI, diosgenin and platycodon, which inhibit osteoclastogenesis and stimulate osteoblast differentiation (Folwarczna et al. 2016; Gu et al. 2016; Jeong et al. 2010; Niu et al. 2011). Based on the reported effect of TEN on the inhibition of osteoclastogenesis, we explored its effect on BMSC osteogenic differentiation and bone formation. The results of the present study showed that TEN promoted osteoblast differentiation, which is similar to data obtained with other saponin compounds. These results suggest that TEN could be a novel compound for the

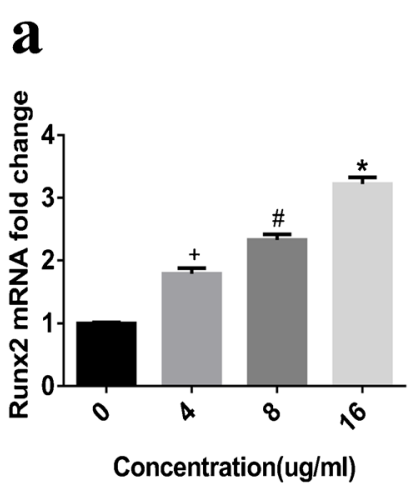

b
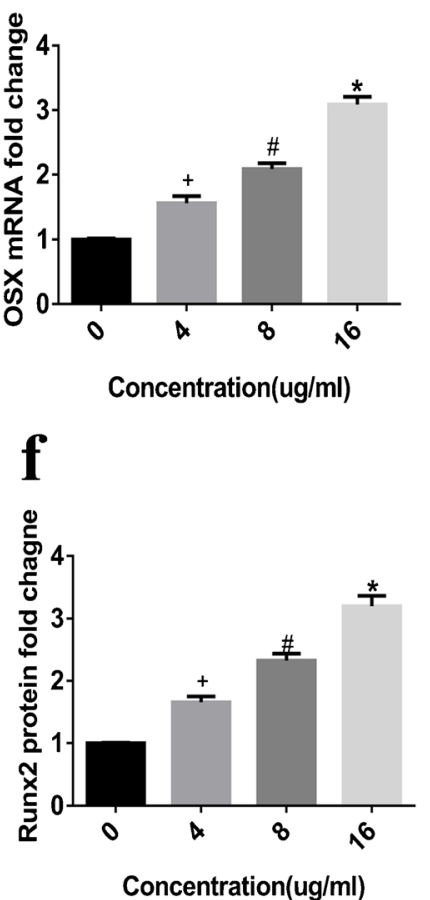

Fig. 3 TEN prevents bone loss in OVX mice. a-a" HE staining of femur sections was assessed after 3 months of treatment. b-b", c-c" The femurs were scanned with a high-resolution X-ray micro-CT and the microstructural indices were performed with the micro-CT data as described in "Materials and methods", including trabecular number (f), trabecular thickness $(\mathbf{g})$ and trabecular BMD $(\mathbf{h})$. Body weight $(\mathbf{d})$ and uterine weight (e) were also performed to confirm the effect of the ovarian excision. Scale bars (a-a") $50 \mu \mathrm{m},(\mathbf{b}-\mathbf{b} ") 1 \mathrm{~mm},(\mathbf{c}-\mathbf{c} ") 100 \mu \mathrm{m}$. Data are shown as mean \pm SD from at least three independent experiments, $* P<0.05$

modulation of osteoblast differentiation and indicate a need for continued study of TEN as a potential therapeutic agent for

c

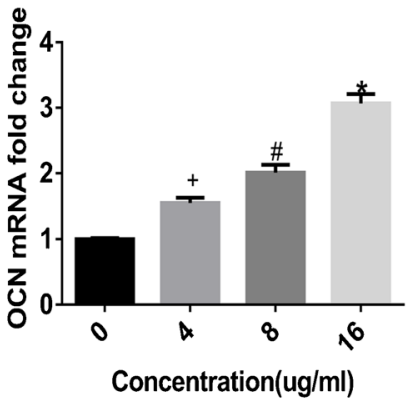

g

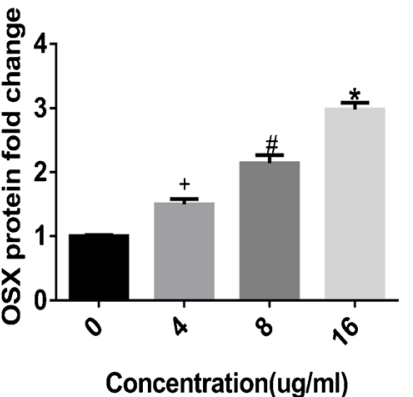

d

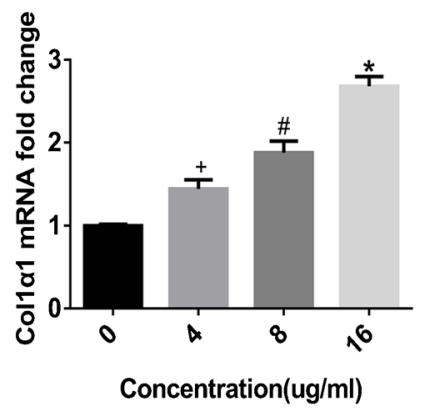

h

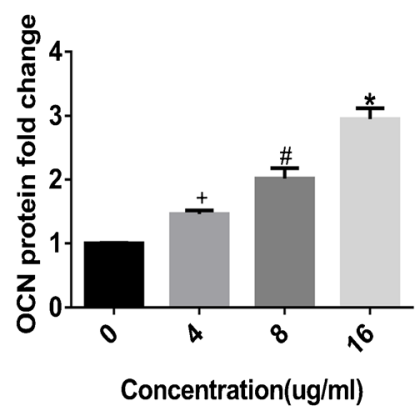

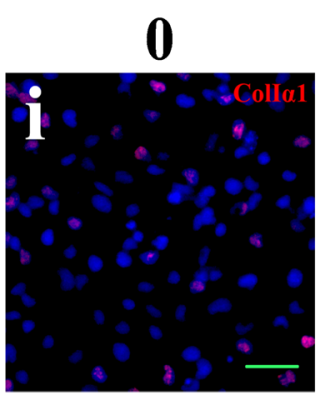
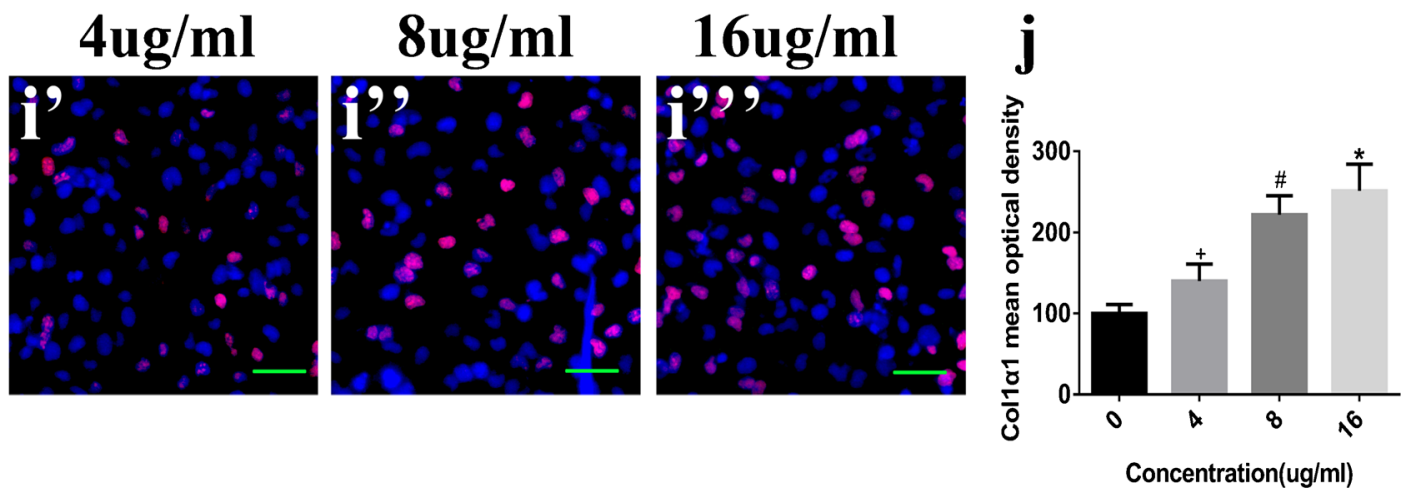

Fig. 2 Effect of TEN on osteogenic markers in vitro. BMSCs were cultured in osteogenic medium and exposed to TEN $(4,8,16 \mu \mathrm{g} / \mathrm{ml})$ for 14 days. Total cytosolic RNA was prepared and used for quantitative RT polymerase chain reaction analysis of Runx2 (a), OSX (b), OCN (c) and Col I $\alpha 1$ (d) as described in "Materials and methods".
Cell lysates $(20 \mu \mathrm{g})$ were obtained for western blot and analyzed using antibodies specific for Runx2 (e, f), OSX (e, g) and OCN (e, h). The expression of Col $\mathrm{I} \alpha 1$ was assessed by immunofluorescence staining $(\mathbf{i}-\mathbf{i}$ "', j). Scale bar $5 \mu \mathrm{m}$. Columns represent means \pm SD from 3 independent experiments, each performed in triplicate 

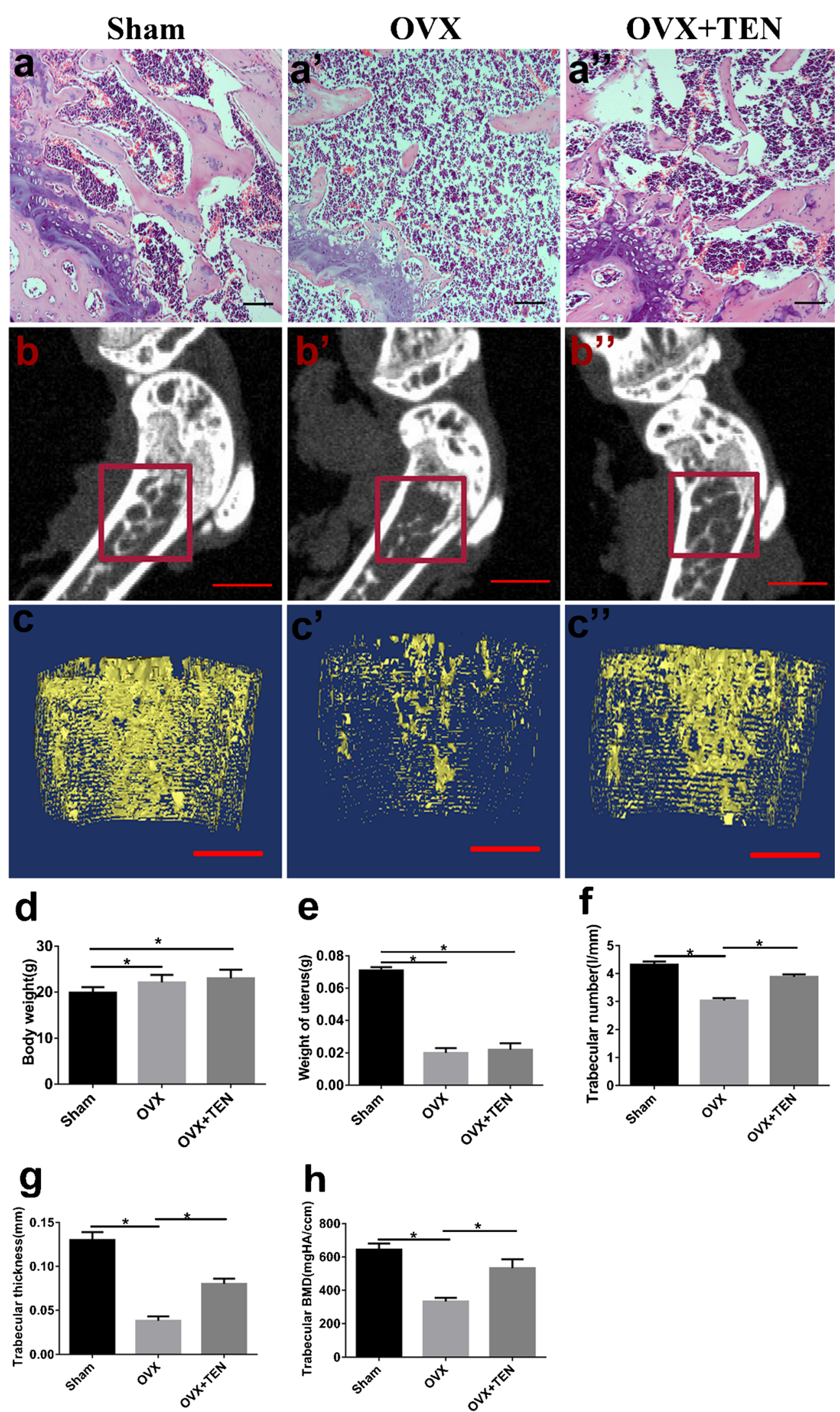

bone disorders, since it is expected to not only enhance bone formation but also suppress bone resorption.
Osteogenic differentiation is a complex process that involves a large number of regulators such as ALP, Ruxn2, 
Fig. 4 Effect of TEN on the expression of Runx2, OSX and $\mathrm{OCN}$ in vivo. Weak positive staining for Runx2 (a-a", d), $\operatorname{OSX}(\mathbf{b}-\mathbf{b} ", \mathbf{e})$ and OCN $(\mathbf{c}-\mathbf{c}$ ", f) was observed, especially on the surface of bone lacuna in the OVX group after 3 months. Scale bars (a-a"), (b-b"), (c-c") $20 \mu \mathrm{m}$. Columns represent means \pm SD from at least three independent experiments, $* P<0.05$
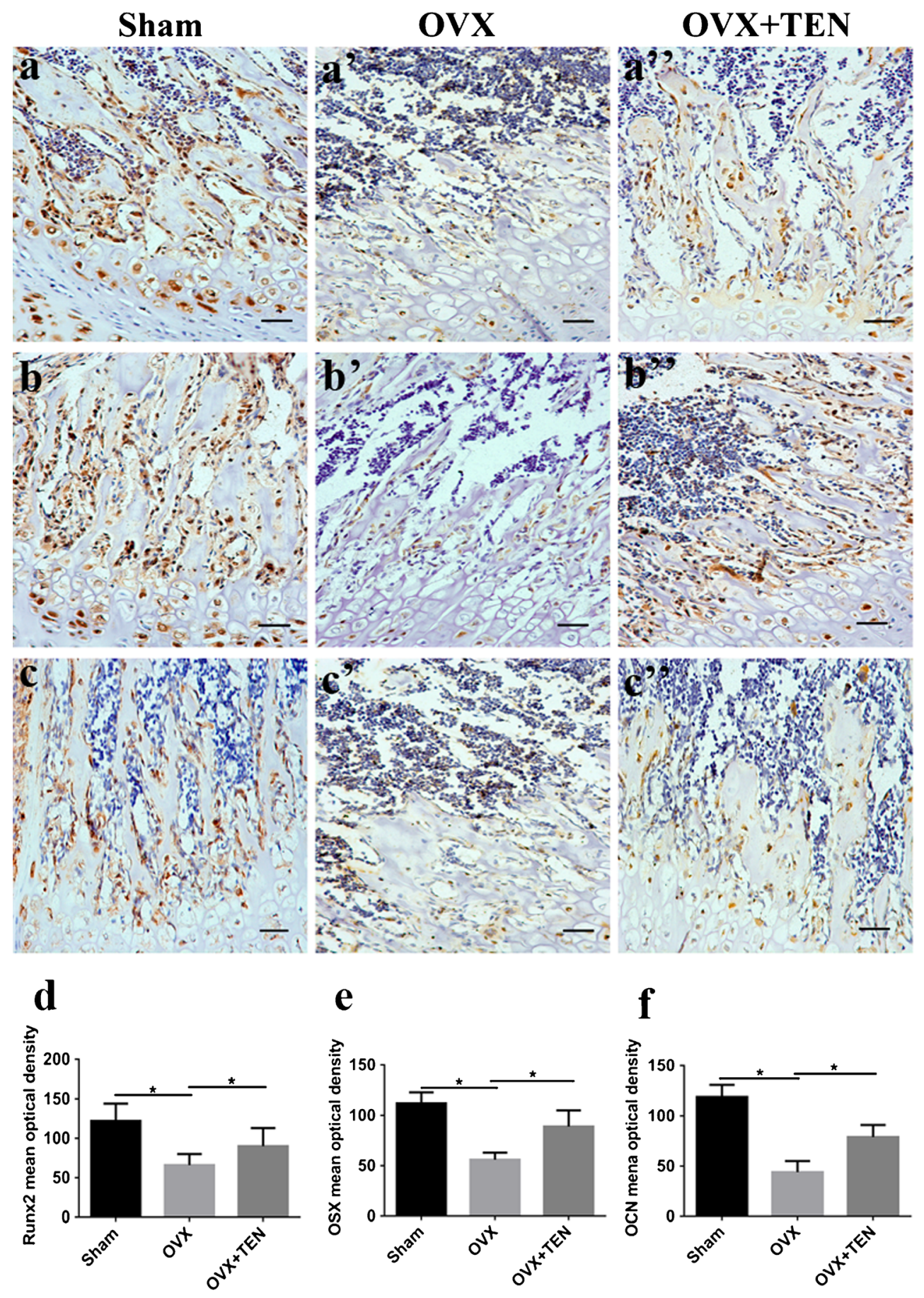

(Lee et al. 2008). OCN is involved in controlling the mineralization process; it appears at a late stage of osteogenic differentiation and is expressed in mature cells of the osteoblastic lineage (Sun et al. 2010; Hauschka et al. 1989). Col I $\alpha 1$, an important component of the bone extracellular matrix, connects the cell surface integrins with other extracellular matrix proteins (Lian and Stein 1992). In the present study, TEN effectively upregulated ALP, Runx2, OSX, OCN and Col I $\alpha 1$, thereby enhancing tion and modulate the expression of other bone markers 


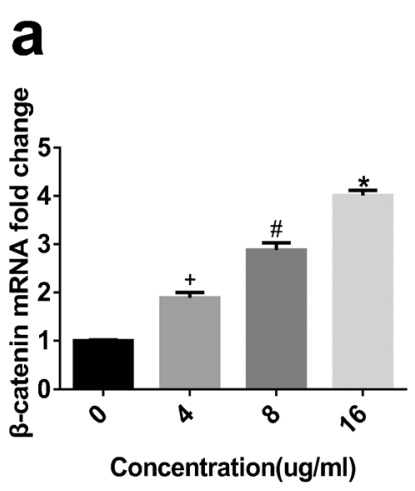

b
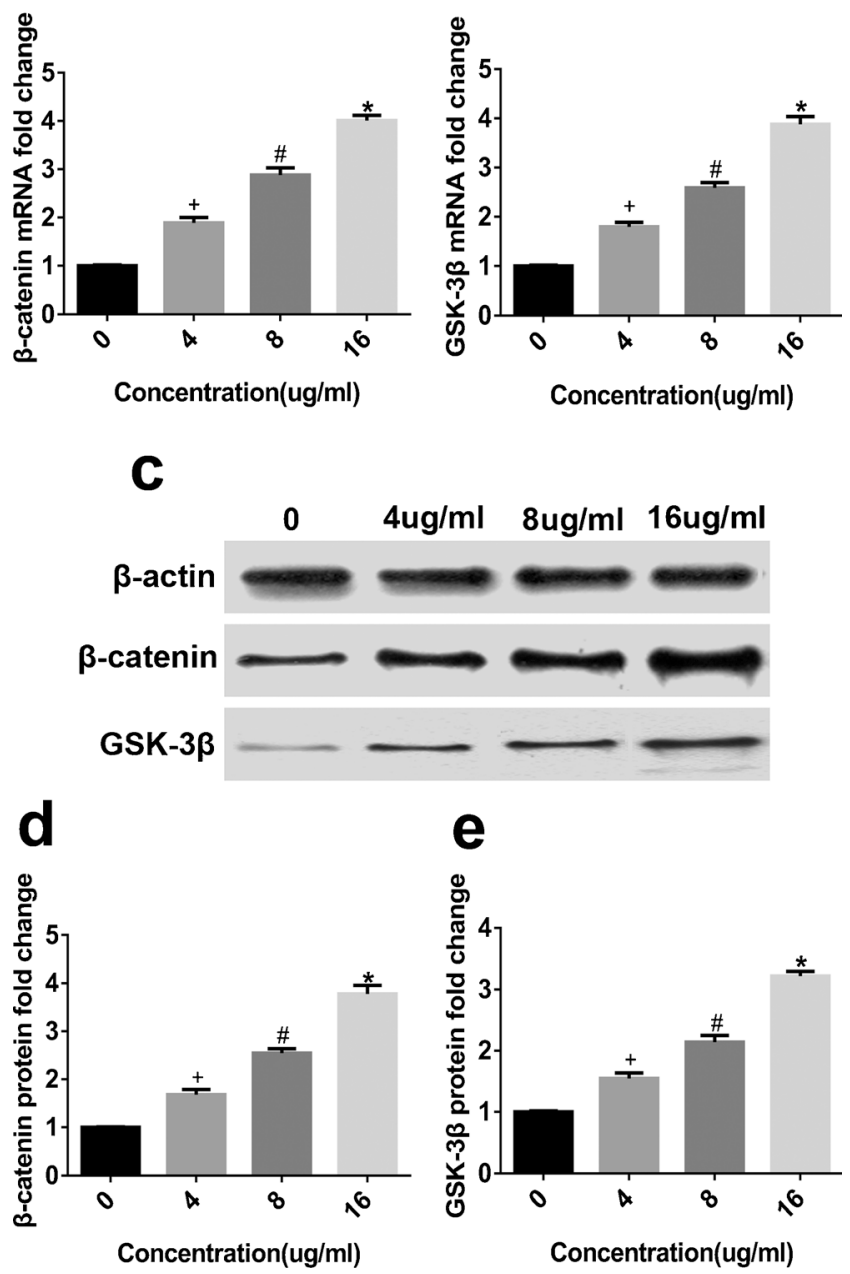

Fig. 5 Effect of TEN on WNT/ $\beta$-catenin signaling. BMSCs were cultured in osteogenic differentiation medium with the treatment of TEN (4, $8,16 \mathrm{ug} / \mathrm{ml}$ ) for 14 days. The mRNA expression of $\beta$-catenin (a) and GSK-3 $\beta$ (b) was measured by Q-PCR. The protein expression of $\beta$ catenin $(\mathbf{c}, \mathbf{d})$ and GSK-3 $\beta$ (c, e) was assessed by western blot. ${ }^{+} P<0.05$ veruss group without TEN, ${ }^{\#} P<0.05$ compared with $4 \mu \mathrm{g} / \mathrm{ml}$ TEN, $* P<0.05$ compared with $8 \mu \mathrm{g} / \mathrm{ml}$ TEN. Columns represent mean \pm SD from 3 independent experiments, each performed in triplicate

mineralization. TEN promoted the osteogenic differentiation and maturation of BMSCs, suggesting that TEN is effective in preventing osteoporosis by promoting BMSC function.

To investigate the effects of TEN on postmenopausal osteoporosis in vivo, we used the OVX mice model (Spilmont et al. 2013). Our data showed that TEN significantly improved the trabecular bone microarchitecture of the distal femur in OVX mice. This suggests that TEN is effective in preventing OVX-induced bone loss. These findings were confirmed by histological examination of femur tissues using HE staining and by assessing the expression of Runx2, OSX and OCN in femurs. Consistent with the in vitro results, TEN upregulated osteogenic markers
Tenuigenin

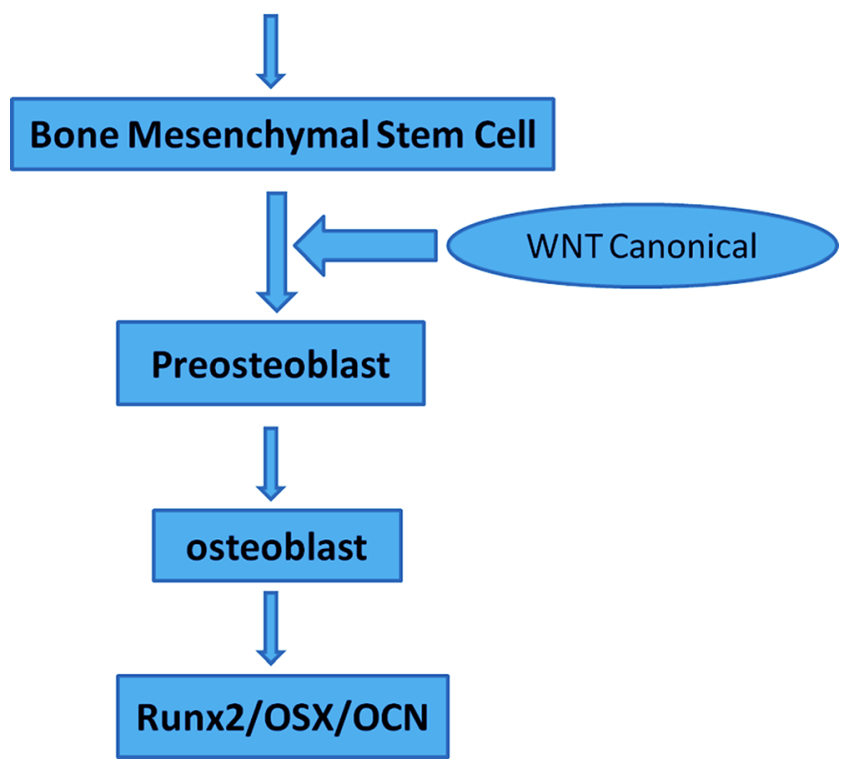

Fig. 6 TEN promotes differentiation of BMSCs into osteoblasts. The maturation and survival of osteoblasts are supported by WNT canonical signaling, resulting in the enhancement of Runx2, OSX and OCN

in vivo, indicating that TEN stimulates bone formation and growth. Taken together, our in vitro and in vivo results show that TEN not only promoted osteogenic differentiation in the early stage but also in the late stage of the osteogenic process, which is similar to the effects of other saponin compounds such as ginsenoside, asperosaponin VI, diosgenin and platycodon.

Several studies have reported that osteoporosis in postmenopausal women is often accompanied by depression, neurasthenia and hypomnesis (Gonzalez-Rodriguez et al. 2016; Heuser and Staemmler 1973; Hickey et al. 2016). TEN has been shown to ameliorate learning and memory impairments induced by ovariectomy (Cai et al. 2013). As TEN has anti-aging, anti-depressant, neuroprotective and neuroregene rative effects, it may be more effective than other saponin compounds. TEN is suitable for the treatment of women with osteoporosis associated with depression, neurasthenia, hypomnesis, memory impairments and many other degenerative diseases.

$\mathrm{WNT} / \beta$-catenin signaling, which is a crucial signal pathway, has sparked great interest in recent decades for its essential role in regulating bone biology and disease ( $\mathrm{Gu}$ et al. 2015; Manolagas 2014; Tao et al. 2016). A previous study reported that WNT signaling can directly suppress the commitment of MSCs to chondrogenic and adipogenic lineages and enhance osteogenic differentiation via both $\beta$-catenin-dependent and $\beta$-catenin-independent mechanisms (Hill et al. 2005). Moreover, the expression of the markers of 
mature osteoblasts ALP, OSX, Col I $\alpha 1$, Runx2 and OCN in osteoblasts can be increased by activation of the WNT/ß-catenin pathway (Frey et al. 2015; Hwang et al. 2015; Li et al. 2015). To gain further insight into TEN-induced osteogenic differentiation, we investigated the expression of $\beta$-catenin and GSK3 $\beta$, which are key factors in WNT signaling that regulate bone formation (Rossini et al. 2013). Our results showed that $\beta$-catenin and GSK $3 \beta$ were upregulated by TEN, suggesting that TEN stimulates BMSC osteogenic differentiation by activating WNT signaling.

The present study had several limitations. First, the effect of TEN on rat BMSCs was examined under osteogenic differentiation conditions. The effect of TEN on human BMSCs needs to be examined to substantiate these results. Second, although our findings indicate a protective effect of TEN on osteoporotic bone, we did not include a positive control group, such as estrogen treatment or a control+TEN group. A control group, control+TEN group and sham+ estrogen group are worthy of further in-depth study. Third, we only obtained BMSCs from mice because of ethical reasons. Additional human BMSCs from healthy persons and osteoporosis patients would be helpful to identify whether these findings are specific.

In conclusion, our study provides novel insights into the effects of TEN on BMSC osteogenic differentiation and its protective effect on bone loss in mice. We demonstrated, for the first time, that TEN promotes osteogenesis by upregulating Runx2, OSX and OCN at the tissue and cellular levels, thereby promoting the lineage differentiation of BMSCs into osteoblasts. Furthermore, we have shown that WNT signaling is at least partly involved as an underlying mechanism in this process (Fig. 6). In future studies, the use of TEN for the stimulation of bone formation in postmenopausal women and the treatment of depression, neurasthenia, hypomnesis, memory impairments and many other degenerative diseases should be examined by performing pharmacokinetic and toxicological analyses in humans as well as randomized control studies.

Acknowledgments The authors thank PingLing Lai (Medical Experiment Laboratory, The Third Affiliated Hospital, Southern Medical University, Guangzhou, Guangdong, People's Republic of China) for excellent technical support with X-ray $\mu \mathrm{CT}$. This work was supported by the National Natural Science Foundation of China (31370985) and the National Science Foundation of Guangdong Province of China (2015A030310416).

\footnotetext{
Author contributions All authors made substantial contributions to conception and design, acquisition of data and interpretation of data; took part in either drafting the article or revising it critically for vital intellectual content; gave final approval of the version to be published; and agreed to be accountable for all aspects of the work in ensuring that questions related to the accuracy or integrity of any part of the work are appropriately investigated and resolved.
}

\section{Compliance with ethical standards}

Disclosure The authors report no conflicts of interest in this work.

Open Access This article is distributed under the terms of the Creative Commons Attribution 4.0 International License (http:// creativecommons.org/licenses/by/4.0/), which permits unrestricted use, distribution, and reproduction in any medium, provided you give appropriate credit to the original author(s) and the source, provide a link to the Creative Commons license, and indicate if changes were made.

\section{References}

Bagan J, Peydro A, Calvo J, Leopoldo M, Jimenez Y, Bagan L (2016) Medication-related osteonecrosis of the jaw associated with bisphosphonates and denosumab in osteoporosis. Oral Dis 22: 324-329

Bianco P, Kuznetsov SA, Riminucci M, Gehron RP (2006) Postnatal skeletal stem cells. Methods Enzymol 419:117-148

Boyle WJ, Simonet WS, Lacey DL (2003) Osteoclast differentiation and activation. Nature 423:337-342

Cai ZL, Wang CY, Gu XY, Wang NJ, Wang JJ, Liu WX, Xiao P, Li CH (2013) Tenuigenin ameliorates learning and memory impairments induced by ovariectomy. Physiol Behav 118:112-117

Demontiero O, Vidal C, Duque G (2012) Aging and bone loss: new insights for the clinician. Ther Adv Musculoskelet Dis 4:61-76

Eriksen EF, Diez-Perez A, Boonen S (2014) Update on long-term treatment with bisphosphonates for postmenopausal osteoporosis: a systematic review. Bone 58:126-135

Folwarczna J, Zych M, Nowinska B, Pytlik M, Bialik M, Jagusiak A, Lipecka-Karcz M, Matysiak M (2016) Effect of diosgenin, a steroidal sapogenin, on the rat skeletal system. Acta Biochim Pol 63:287-295

Frey JL, Li Z, Ellis JM, Zhang Q, Farber CR, Aja S, Wolfgang MJ, Clemens TL, Riddle RC (2015) Wnt-Lrp5 signaling regulates fatty acid metabolism in the osteoblast. Mol Cell Biol 35:1979-1991

Gonzalez-Rodriguez, A., Catalan, R., Penades, R., and Bernardo, M. (2016). The oestrogen dysfunction hypothesis in schizophrenia: The need for an integrative approach to treat postmenopausal women. Aust N Z J Psychiatry (in press)

Gu Q, Chen C, Zhang Z, Wu Z, Fan X, Zhang Z, Di W, Shi L (2015) Ginkgo biloba extract promotes osteogenic differentiation of human bone marrow mesenchymal stem cells in a pathway involving Wnt/ beta-catenin signaling. Pharmacol Res 97:70-78

Gu Y, Zhou J, Wang Q, Fan W, Yin G (2016) Ginsenoside Rg1 promotes osteogenic differentiation of rBMSCs and healing of rat tibial fractures through regulation of GR-dependent BMP-2/SMAD signaling. Sci Rep 6:25282

Harada S, Rodan GA (2003) Control of osteoblast function and regulation of bone mass. Nature 423:349-355

Hauschka PV, Lian JB, Cole DE, Gundberg CM (1989) Osteocalcin and matrix Gla protein: vitamin K-dependent proteins in bone. Physiol Rev 69:990-1047

Heuser HP, Staemmler HJ (1973) Histological investigations into the effect of oestriol succinate on the corpus uteri in postmenopausal women. Arzneimittelforschung 23:558-562

Hickey, M., Schoenaker, D.A., Joffe, H., and Mishra, G.D. (2016). Depressive symptoms across the menopause transition: findings from a large population-based cohort study. Menopause (in press)

Hill TP, Spater D, Taketo MM, Birchmeier W, Hartmann C (2005) Canonical Wnt/beta-catenin signaling prevents osteoblasts from differentiating into chondrocytes. Dev Cell 8:727-738 
Hwang JH, Cha PH, Han G, Bach TT, Min DS, Choi KY (2015) Euodia sutchuenensis Dode extract stimulates osteoblast differentiation via Wnt/beta-catenin pathway activation. Exp Mol Med 47:e152

Jeong HM, Han EH, Jin YH, Hwang YP, Kim HG, Park BH, Kim JY, Chung YC, Lee KY, Jeong HG (2010) Saponins from the roots of Platycodon grandiflorum stimulate osteoblast differentiation via $\mathrm{p} 38$ MAPK- and ERK-dependent RUNX2 activation. Food Chem Toxicol 48:3362-3368

Karsenty G (2003) The complexities of skeletal biology. Nature 423:316-318

Kronenberg HM (2003) Developmental regulation of the growth plate. Nature 423:332-336

Lee HW, Suh JH, Kim HN, Kim AY, Park SY, Shin CS, Choi JY, Kim JB (2008) Berberine promotes osteoblast differentiation by Runx2 activation with p38 MAPK. J Bone Miner Res 23:1227-1237

Li X, Lim J, Lu J, Pedego TM, Demer L, Tintut Y (2015) Protective Role of Smad6 in Inflammation-Induced Valvular Cell Calcification. J Cell Biochem 116:2354-2364

Li F, Zhou C, Xu L, Tao S, Zhao J, Gu Q (2016) Effect of Stem Cell Therapy on Bone Mineral Density: A Meta-Analysis of Preclinical Studies in Animal Models of Osteoporosis. PLoS ONE 11, e149400

Lian JB, Stein GS (1992) Concepts of osteoblast growth and differentiation: basis for modulation of bone cell development and tissue formation. Crit Rev Oral Biol Med 3:269-305

Liang Z, Shi F, Wang Y, Lu L, Zhang Z, Wang X, Wang X (2011) Neuroprotective effects of tenuigenin in a SH-SY5Y cell model with 6-OHDA-induced injury. Neurosci Lett 497:104-109

Liu W, Yang LH, Kong XC, An LK, Wang R (2015) Meta-analysis of osteoporosis: fracture risks, medication and treatment. Minerva Med 106:203-214

Luhe A, Kunkele KP, Haiker M, Schad K, Zihlmann C, Bauss F, Suter L, Pfister T (2008) Preclinical evidence for nitrogencontaining bisphosphonate inhibition of farnesyl diphosphate (FPP) synthase in the kidney: implications for renal safety. Toxicol In Vitro 22:899-909

Ma ZP, Liao JC, Zhao C, Cai DZ (2015) Effects of the 1, 4dihydropyridine L-type calcium channel blocker benidipine on bone marrow stromal cells. Cell Tissue Res 361:467-476

Manolagas SC (2014) Wnt signaling and osteoporosis. Maturitas 78:233237

Niu Y, Li Y, Huang H, Kong X, Zhang R, Liu L, Sun Y, Wang T, Mei Q (2011) Asperosaponin VI, a saponin component from Dipsacus asper wall, induces osteoblast differentiation through bone morphogenetic protein-2/p38 and extracellular signal-regulated kinase 1/2 pathway. Phytother Res 25:1700-1706

Rodan GA, Martin TJ (2000) Therapeutic approaches to bone diseases. Science 289:1508-1514

Rossini M, Gatti D, Adami S (2013) Involvement of WNT/betacatenin signaling in the treatment of osteoporosis. Calcif Tissue Int 93:121-132

Safer, U., Safer, V.B., Demir, S.O., and Yanikoglu, I. (2016). Effects of Bisphosphonates and Calcium plus Vitamin-D Supplements on Cognitive Function in Postmenopausal Osteoporosis. Endocr Metab Immune Disord Drug Targets (in press)
Serigano K, Sakai D, Hiyama A, Tamura F, Tanaka M, Mochida J (2010) Effect of cell number on mesenchymal stem cell transplantation in a canine disc degeneration model. J Orthop Res 28:1267-1275

Shin KY, Won BY, Ha HJ, Yun YS, Lee HG (2015) Genotoxicity studies on the root extract of Polygala tenuifolia Willdenow. Regul Toxicol Pharmacol 71:365-370

Spilmont M, Leotoing L, Davicco MJ, Lebecque P, Mercier S, MiotNoirault E, Pilet P, Rios L, Wittrant Y, Coxam V (2013) Pomegranate seed oil prevents bone loss in a mice model of osteoporosis, through osteoblastic stimulation, osteoclastic inhibition and decreased inflammatory status. J Nutr Biochem 24:1840-1848

Sun GB, Deng XC, Li CH (2007) The protective effects of tenuigenin on the PC12 cells injury induced by H2O2. Zhong Yao Cai 30:991-993

Sun H, Feng K, Hu J, Soker S, Atala A, Ma PX (2010) Osteogenic differentiation of human amniotic fluid-derived stem cells induced by bone morphogenetic protein-7 and enhanced by nanofibrous scaffolds. Biomaterials 31:1133-1139

Tao K, Xiao D, Weng J, Xiong A, Kang B, Zeng H (2016) Berberine promotes bone marrow-derived mesenchymal stem cells osteogenic differentiation via canonical Wnt/beta-catenin signaling pathway. Toxicol Lett 240:68-80

van den Bergh JP, van Geel TA, Geusens PP (2012) Osteoporosis, frailty and fracture: implications for case finding and therapy. Nat Rev Rheumatol 8:163-172

Xu YX, Wu CL, Wu Y, Tong PJ, Jin HT, Yu NZ, Xiao LW (2012) Epimedium-derived flavonoids modulate the balance between osteogenic differentiation and adipogenic differentiation in bone marrow stromal cells of ovariectomized rats via Wnt/beta-catenin signal pathway activation. Chin J Integr Med 18:909-917

Yang S, Li X, Cheng L, Wu H, Zhang C, Li K (2015) Tenuigenin inhibits RANKL-induced osteoclastogenesis by down-regulating NFkappaB activation and suppresses bone loss in vivo. Biochem Biophys Res Commun 466:615-621

Ying X, Sun L, Chen X, Xu H, Guo X, Chen H, Hong J, Cheng S, Peng L (2013) Silibinin promotes osteoblast differentiation of human bone marrow stromal cells via bone morphogenetic protein signaling. Eur J Pharmacol 721:225-230

Yonezawa T, Lee JW, Hibino A, Asai M, Hojo H, Cha BY, Teruya T, Nagai K, Chung UI, Yagasaki K et al (2011) Harmine promotes osteoblast differentiation through bone morphogenetic protein signaling. Biochem Biophys Res Commun 409:260-265

Yu GY, Zheng GZ, Chang B, Hu QX, Lin FX, Liu DZ, Wu CC, Du SX, Li XD (2016) Naringin Stimulates Osteogenic Differentiation of Rat Bone Marrow Stromal Cells via Activation of the Notch Signaling Pathway. Stem Cells Int 2016:7130653

Zhang LY, Xue HG, Chen JY, Chai W, Ni M (2016) Genistein induces adipogenic differentiation in human bone marrow mesenchymal stem cells and suppresses their osteogenic potential by upregulating PPARgamma. Exp Ther Med 11:1853-1858

Zou L, Zou X, Chen L, Li H, Mygind T, Kassem M, Bunger C (2008) Multilineage differentiation of porcine bone marrow stromal cells associated with specific gene expression pattern. J Orthop Res 26: $56-64$ 